\title{
Photon management for photovoltaics
}

\author{
E.T. Yu and J. van de Lagemaat, Guest Editors
}

Photovoltaics are expected to play an important role in the future energy infrastructure. However, achieving simultaneously high efficiency in both light absorption and carrier collection remains a challenging tradeoff. Photon management, which refers to the engineering of materials and device structures to control the spatial distribution of optical energy, offers a number of promising routes to optimizing this tradeoff. Progress in fabrication of nanostructured materials combined with advances in the understanding of nanophotonic devices has enabled new strategies for photon management in a range of photovoltaic devices. Prominent among these are structures with pronounced surface topography or graded refractive-index profiles that reduce surface reflectivity; materials processing that increases optical absorption in materials such as silicon; incorporation of semiconductor nanostructures that enables simultaneous improvements in optical absorption and photogenerated carrier collection; and coherent light trapping in optical waveguide modes via plasmonic or optical scattering effects. The articles in this issue review some of these emerging directions.

\section{Background}

World events and an increasing realization of the importance of clean, renewable sources of energy have fueled intense interest and activity in the development and implementation of a broad range of green energy technologies. Solar energy, including photovoltaic energy conversion, has enjoyed a prominent position within the green energy portfolio. In addition, sustained progress in mainstream solar energy technologies as well as revolutionary breakthroughs over the last two decades in the fabrication, understanding, and application of solid-state and soft-material nanostructures have reignited both commercial and research interest in photovoltaic devices. However, several challenges must be overcome for the tantalizing promise of photovoltaics to be fully realized. Among these, the fundamental tradeoff between light absorption and collection of photogenerated electrons and holes ranks as one of the most important. The engineering of materials and device structures to achieve new levels of control over photon propagation and light energy distribution in photovoltaic devices and systems"photon management" - has emerged as a powerful approach for overcoming this tradeoff while also reducing reflectivity losses and enabling photovoltaic functionality to be achieved in new device and system-level form factors.

The articles in this issue of MRS Bulletin address a number of directions and recent advances in this rapidly developing field. Traditional approaches to photon management in photovoltaics have involved the use of antireflection coatings or surface texturing to maximize transmission of incident light into, or photon path length within, a solar cell; design of device structures in which absorption is maximized in regions for which the effects of minority carrier recombination are minimized; and integration with optical concentrators to direct sunlight incident over a large area onto a small, high-efficiency, high-value solar cell. More recently, a number of new directions for photon management have emerged in which subwavelength structures are employed to control, in a wavelength-dependent manner, photon propagation into and within photovoltaic devices. A defining feature of these approaches is that they exploit the wavenature of light and are therefore able to surpass classical limits of light confinement based on surface texturing. They allow for the use of thinner active layers or structures that are optimized for charge collection and potentially lower the cost of photovoltaic devices or increase their conversion efficiency.

\section{Improving light collection}

The high refractive index $n$ of inorganic semiconductor materials leads to high optical reflectivity at a direct, planar interface between such a semiconductor and a low-index medium (e.g., air or glass), as shown schematically in Figure 1a. Since reflected light is not available for conversion to electricity in a solar cell, approaches for reducing this reflectivity are an essential element of photon management in photovoltaic devices. 


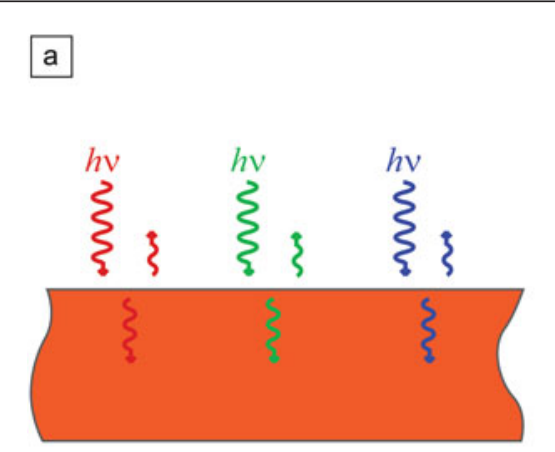

b

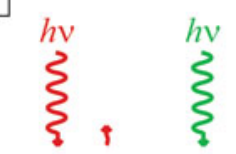

$h \mathrm{v}$
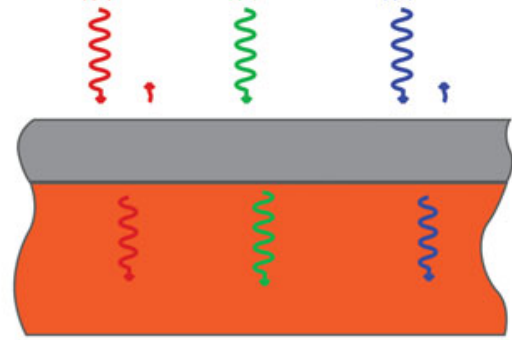

C

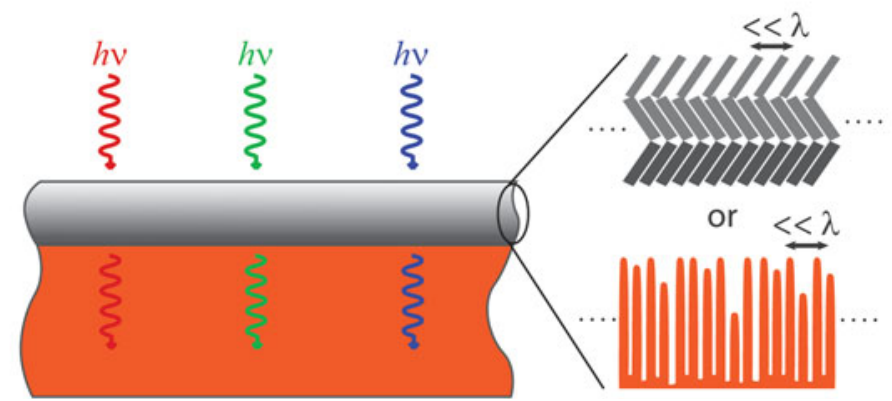

Figure 1. Reducing light reflection in photovoltaics. (a) At a planar interface between air (or glass) and a typical semiconductor photovoltaic material, a substantial fraction of incident light, represented by photons labeled $h v$, is reflected due to the large mismatch in refractive index at the interface. (b) A dielectric film with appropriately chosen thickness and refractive index can eliminate reflection of light at a selected wavelength (for normally incident light) and reduce reflection over a broad range of wavelengths. (c) A dielectric film with a continuous or stepwise grading in refractive index-close to that of air or glass, as appropriate, at the top interface and approaching that of the underlying semiconductor material at the bottom - can act as a highly effective antireflection coating over the broad range of wavelengths and incident angles of light required for photovoltaic applications.

processing of materials have made possible the realization of graded-index coatings via the creation of surface structures with features at length scales comparable to or smaller than the wavelength of incident light, and shapes that effectively provide a continuous or finely stepped increase in refractive index from that of air to that of a photovoltaic material such as silicon. ${ }^{5-8}$ This concept is illustrated schematically in Figure 1c.

In one such approach, described by Poxson et al. in this issue, angled deposition of dielectric films results in the creation of subwavelengthscale nanorods within the film; the effective refractive index of such a film is reduced from that of the bulk material by a factor equal to the fractional volume occupied by the rods, allowing effective refractive indices lower than that of any available bulk dielectric material, and approaching that of air, to be obtained. By controlling deposition conditions and materials to obtain a stepwise gradation in refractive index, low surface reflectivity over a broad range of wavelengths relevant to photovoltaics, and for a wide range of incident angles, can be obtained. ${ }^{9,10}$

For crystalline silicon, which constitutes the largest portion of the current market for photovoltaics, an alternate means to realize an effective grading in refractive index and consequently a low-reflectivity surface involves the use of metal-catalyzed etching to create rod- or cone-

Traditionally, this has often been accomplished via incorporation of a thin-film antireflection coating on the photovoltaic device surface, as illustrated in Figure 1b. However, because such coatings typically rely, in part, upon interference effects to minimize reflectivity, achieving low reflectivity of incident light over a broad range of wavelengths, angles of incidence, and polarizations - as would be desired for photovoltaics - is problematic even with complex (and often expensive) multilayer coatings. ${ }^{1}$

Recent advances in the creation of nanostructures on solidstate surfaces have made possible a variety of additional approaches for reducing the surface reflectivity of photovoltaic devices. It is well known that a graded-index coating, in which a continuous or finely stepped increase in refractive index from an external medium (e.g., air) to the photovoltaic device surface is implemented over a length scale of a few optical wavelengths, can provide highly effective anti-reflection functionality over a broad range of wavelengths and incident angles..$^{2-4}$ This occurs because there is no longer any individual interface from which light can reflect; instead, light smoothly transitions from one refractive index to the other. The lack of materials with refractive indices approaching that of air has traditionally impeded realization of such coatings. Over the past several years, however, advances in deposition and surface like subwavelength structures on the silicon surface. Subwavelength nanostructures are needed because for more macroscopic structures, the effective refractive index would not be homogenous, and strong reflections and light scattering would occur. These surface structures can be designed (e.g., using cones with a bigger base at the largest depth) to lead to a nearly continuous increase of the volume filling fraction of silicon material with depth. Because the index of refraction is a function of the amount of silicon versus air, this in turn leads to the desired smooth transition from a low refractive index equal to that of air to that of silicon, and therefore to an ultralow-reflectivity surface. ${ }^{11}$ This approach has been exploited to create low-reflectivity surfaces on crystalline silicon solar cells with demonstrated solar to electrical power conversion efficiencies as high as $16.8 \% .^{12}$

Trapping of light within a photovoltaic device can enable improvement in both collection and absorption of incident photons, the latter by effectively increasing the path length of photons within the absorbing medium. Micrometer-scale surface texturing, illustrated schematically in Figure 2a and 2b, randomizes the direction of light within the device volume and, with sufficiently high aspect ratios, can enable improved light collection by creating multiple interactions of incident photons with the device surface. Surface texturing can increase optical absorption in a weakly absorbing material by up to a factor 

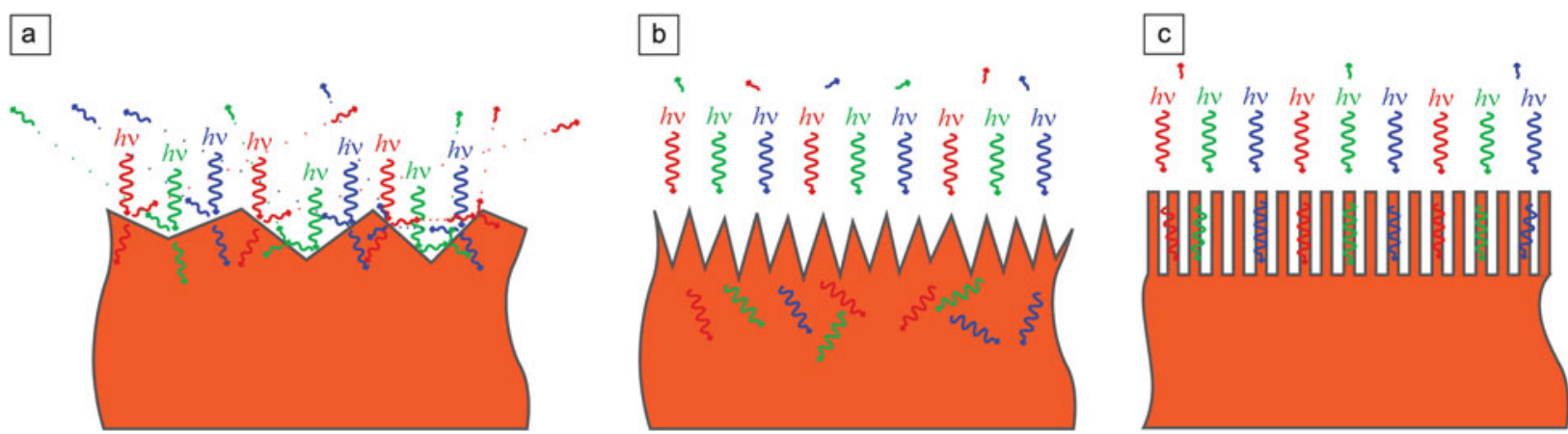

Figure 2. Trapping light in a photovoltaic device. (a) For bulk semiconductor photovoltaic devices (e.g., mono- or polycrystalline silicon), traditional surface texturing provides improved collection and trapping of light in the device, as surface topography enables incident light to undergo multiple reflections with increased total probability of transmission into the device. Deposition of an antireflection coating, as in Figure 1b, on the textured surface can further improve light collection and trapping efficiency. The optical path length for light in the device is also increased, which can result in improved optical absorption efficiency as well. (b) Submicron-scale surface texturing, as in laser-processed "black" silicon, can also be highly effective in light trapping for photovoltaics. (c) Nanowire-based photovoltaic device structures that consist of subwavelength-scale arrays of wires can trap light through a combination of multiple-reflection and near-field optical effects.

of $4 n^{2},{ }^{13,14}$ corresponding to an increase of approximately $50 \times$ for a material such as crystalline silicon. Starting in the late 1990s, techniques based on ultrafast laser processing have been applied to create micrometer-scale to subwavelength surface structures on silicon and other semiconductor materials to achieve low reflectivity. ${ }^{15-18}$ The use of ultrafast laser processing to create micrometer-scale variations in surface morphology and consequently improve light collection is described in the article by Sher et al. in this issue.

\section{Light absorption and carrier collection}

The ultrafast laser processing approach described previously has also been employed for direct engineering of optical absorption in materials - specifically silicon. Femtosecond laser-induced incorporation of very high densities of impurities such as sulfur into silicon has been shown to lead to dramatic increases in optical absorption (see Figure 4 in the article by Sher et al. in this issue), including absorption at energies below the silicon bandgap, leading to an increase in spectral responsivity and therefore solar cell efficiency. In their article, Sher et al. discuss some of the issues that arise in the exploitation of these effects to improve power conversion efficiency in silicon-based photovoltaics.

Surface nanostructuring extended to encompass the entire solar cell volume can also enable improved absorption of incident sunlight via trapping of light within the photovoltaic device, as illustrated schematically in Figure 2c. For solid-state photovoltaic devices, nanowire-based geometries can offer improved light collection (low reflectivity) and absorption via a combination of multiple reflections and increased concentration of light energy within the semiconductor material. ${ }^{19-23}$ But while nanostructuring of the active photovoltaic device material can enable improved light collection and absorption, there is often a tradeoff in device performance because of increased surface recombination of photogenerated electrons and holes that can occur due to defects that are introduced into the material by the nanostructuring process. Indeed, a fundamental challenge for many photovoltaic device technologies is achieving, simultaneously, high efficiency in both broadband optical absorption and collection of the resulting photogenerated carriers. In this respect, nanowire-based solar cell geometries can offer an additional advantage. For nanowire-based inorganic semiconductor solar cells, creation of $p-n$ junctions in the radial direction of each nanowire can provide photogenerated carriers with a shorter transport path to the appropriate electrode at which they can be collected as electrical current..$^{24,25}$ While this advantage must be weighed against increased surface recombination at the nanowire surface, electrical resistance associated with metal contacts to each nanowire, and added process complexity, ${ }^{26}$ recent reports have suggested that the benefits of light trapping and improved absorption in nanowire-based geometries can in some cases outweigh the detrimental effects of increased recombination. ${ }^{27}$ For devices such as dye-sensitized or hybrid inorganic-organic solar cells, the use of nanowire structures for electron transport has been shown to enable improved performance by providing a more direct path for collection of photogenerated carriers than random nanoparticle networks. ${ }^{28-31}$ The article by Zhu and Frank in this issue addresses the use of nanowire structures for a variety of types of solar cells.

Exploiting the wave nature of light in coherent light trapping approaches can provide additional vehicles for photon management in photovoltaic devices. Recently, Atwater reviewed the use of plasmonic structures as well as photonic crystals for light trapping in photovoltaic structures. ${ }^{61}$ In this issue, Mallick et al. describe work in which patterning of a thin-film photovoltaic device into a two-dimensional photonic crystal structure can lead to coupling of incident photons into confined modes associated with the photonic crystal (Figure 3b), effectively trapping them within the device and enabling a large increase in expected photocurrent response over a broad range 


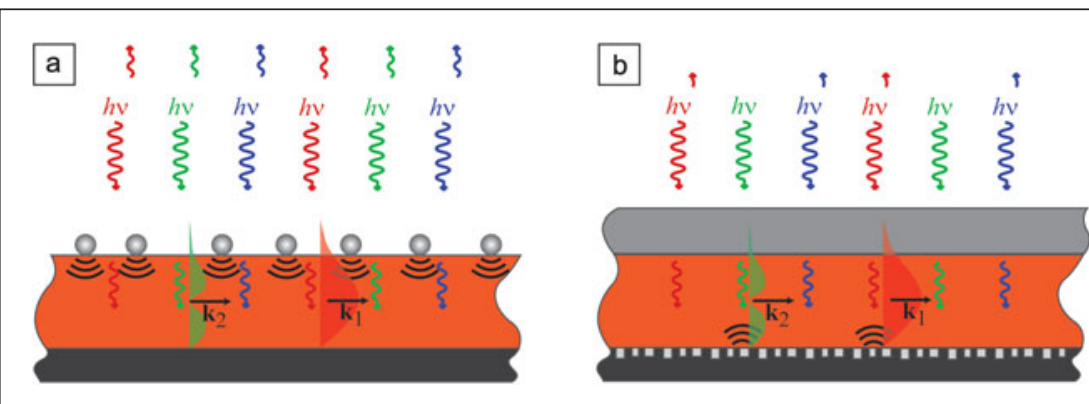

Figure 3. For thin-film photovoltaic devices, micrometer-scale texturing is typically challenging, and alternate approaches for light trapping have emerged. (a) Metal and/ or dielectric nanostructures on the device surface can scatter incident light into optical modes, labeled $\mathbf{k}_{1}$ and $\mathbf{k}_{2}$ in the figure, confined within the thin-film semiconductor device structure. Such scattering can provide photons with dramatically increased path lengths within the device and corresponding increases in optical absorption efficiency. (b) Scattering of light into waveguide modes within a thin-film device can also be accomplished with scattering structures on the back side of the device. This geometry allows scattering to be optimized for longer-wavelength light, for which optical absorption coefficients are typically lower, and enables integration of an antireflection coating on the top surface, as illustrated in the figure.

increase in electromagnetic field amplitude and optical transition probability, which are typically localized to within several nm of the metal nanoparticle in which the plasmonic excitation occurs, can play a prominent role in increasing the photoresponse of an organic photovoltaic device in which metal nanoparticles have been incorporated.

For photovoltaic devices based on inorganic semiconductors, electronic states at metal/ semiconductor interfaces will often lead to nonradiative recombination, and metallic nanostructures embedded within the device would be very likely to degrade overall performance. To exploit plasmonic effects in such devices, metal nanostructures are therefore more commonly located in close proximity to, rather than directly within, the active device structure. Furthermore, optical absorption coefficients are typically lower than in organic materials, and

of wavelengths. They also describe the use of multilayer dielectric stacks and metal nanostructures that accomplish similar coherent light trapping into waveguided modes and discuss the limits of such approaches.

Plasmonics also has emerged as a promising route to advanced photon management in photovoltaics. ${ }^{32,33}$ Plasmons are collective excitations of electrons that occur most prominently in materials with high conduction electron densities, primarily metals. These excitations can occur both in bulk material and, in the case of surface plasmons, at metal surfaces and interfaces. Contrary to the previously discussed strategies, surface plasmons can confine light in spaces significantly shorter than one-fourth of the wavelength of the light and can lead to many orders of magnitude enhancement of the optical fields - a fact exploited in analytical chemistry for surface-enhanced Raman spectroscopy. Because surface plasmon modes are highly sensitive to surface structure and the immediate surrounding dielectric environment, nanostructuring (e.g., to form nanoparticles or asperities), as well as the integration of metals with dielectric materials, can strongly influence their nature. Starting in the mid-1990s, plasmonic absorption and scattering effects in metal nanoparticles either embedded in or deposited atop device structures were explored as potential routes for increasing photocurrent response in organic photovoltaics ${ }^{34-36}$ and silicon-on-insulator photodetectors. ${ }^{37-41}$ More recently, there has been a strong renewal of interest in plasmonic absorption and scattering effects as vehicles for photon management in organic, ${ }^{42,43}$ bulk crystalline, ${ }^{44-46}$ and thin-film photovoltaics and photodetectors. ${ }^{47-57}$ In organic semiconductor photovoltaic devices, relatively large optical absorption coefficients combined with short carrier diffusion lengths mandate that absorption of photons occurs within a very short distance-on the order of $10 \mathrm{~nm}$ - from the donor/acceptor material heterojunction in the device in order to enable efficient photogenerated carrier collection. Thus, plasmonic absorption and the resulting the balance between plasmonic absorption and scattering effects in altering photocurrent response is shifted; while plasmonic absorption in metal nanoparticles and the accompanying field localization effects can still play a significant role, scattering of photons and an accompanying increase in photon path length within the device are generally more prominent. Figure 3 illustrates two approaches in which plasmonic and related scattering effects in metal and dielectric nanostructures are exploited to improve optical absorption in thin-film photovoltaic devices. Catchpole et al.'s article in this issue discusses a variety of issues pertaining to the deployment of plasmonic phenomena and related effects to improve photovoltaic device performance.

A final approach to photon management in photovoltaics, at the system rather than the device level, involves the use of optical concentrators to focus light incident over a large area onto smaller, highly efficient solar cells. Such "concentrating photovoltaic" systems are of increasing commercial interest, and while not addressed directly in this issue, developments in technologies such as microscale ${ }^{58}$ and luminescent concentrators ${ }^{59}$ offer intriguing and promising possibilities for improving the cost-effectiveness of solar energy harvesting, generally.

\section{Prospects}

The obvious abundance and ubiquity of sunlight - full utilization of approximately one hour of sunlight incident on the upper atmosphere of Earth would be sufficient to provide for worldwide energy use for one year - and its ostensibly "free" nature has helped to fuel interest in solar energy as a key contributor to a green energy infrastructure. Despite enormous progress in research and commercial implementation, however, daunting technological, economic, and policy challenges remain, and the proportion of our total energy needs currently met by solar energy harvesting, while growing rapidly in total power generated, remains small and steady in relative size due to the rapid growth of the world's energy use. In the United States, for 
example, only $0.15 \%$ of power was generated by solar photovoltaics in 2010, a number basically equal to that in 2009 and $2008 .^{60}$ It is clear that in order to become a significant part of the energy market and barring major government incentives, the cost of power generation for photovoltaics needs to be significantly reduced. Emerging techniques for photon management in photovoltaics, by bringing light to its optimum location in a solar cell, can help bring the vision of clean, inexpensive energy from the sun a bit closer to reality.

\section{Acknowledgments}

J. vd L. acknowledges support from the Division of Chemical Sciences, Geosciences, and Biosciences, Office of Basic Energy Sciences, U.S. Department of Energy and is an employee of the Alliance for Sustainable Energy, LLC (Alliance), a contractor of the U.S. Government under Contract No. DE-AC3608G028308. E.T.Y. acknowledges partial support from the National Science Foundation (DMR 0806755), the Department of Energy (DE-FG36-08G018016), and the Judson S. Swearingen Regents Chair in Engineering at the University of Texas at Austin.

\section{References}

1. M. Born, E. Wolf, Principles of Optics, 7th Edition (Cambridge University Press, Cambridge, UK, 1999).

2. R.B. Stephens, G.D. Cody, Thin Solid Films 45, 19 (1977).

3. J.I. Gittleman, E.K. Sichel, H.W. Lehmann, R. Widmer, Appl. Phys. Lett. 35, $742(1979)$

4. H.G. Craighead, R.E. Howard, D.M. Tennant, Appl. Phys. Lett. 37, 653 (1980). 5. Z. Yu, H. Gao, W. Wu, H. Ge, S.Y. Chou, J. Vac. Sci. Technol., B 21, 2874 (2003). 6. H. Sai, H. Fujii, K. Arafune, Y. Ohshita, Y. Kanamori, H. Yugami, M. Yamaguchi, Jpn. J. Appl. Phys. 46, 3333 (2007)

7. C.-H. Sun, P. Jiang, B. Jiang, Appl. Phys. Lett. 92, 061112 (2008).

8. H.M. Branz, V.E. Yost, S. Ward, K.M. Jones, B. To, P. Stradins, Appl. Phys. Lett. 94, 231121 (2009)

9. J.Q. Xi, M.F. Schubert, J.K. Kim, E.F. Schubert, M.F. Chen, S.Y. Lin, W. Liu, J.A. Smart, Nat. Photonics 1, 176 (2007).

10. M.L. Kuo, D.J. Poxson, Y.S. Kim, F.W. Mont, L.K. Kim, E.F. Schubert, S.Y. Lin, Opt. Lett. 33, 2527 (2008).

11. S. Koynov, M.S. Brandt, M. Stutzmann, Appl. Phys. Lett. 88, 203107 (2008). 12. H.-C. Yuan, V.E. Yost, M.R. Page, P. Stradins, D.L. Meier, H.M. Branz, Appl. Phys. Lett. 95, 123501 (2009)

13. E. Yablonovitch, J. Opt. Soc. Am. 72, 899 (1982)

14. E. Yablonovitch, G.D. Cody, IEEE Trans. Electron Devices 29, 300 (1982).

15. T.H. Her, R.J. Finlay, C. Wu, S. Deliwala, E. Mazur, Appl. Phys. Lett. 73, 1673 (1998)

16. B.K. Nayak, M.C. Gupta, K.W. Kolasinski, Nanotechnology 18, 195302 (2007) 17. J. Zhao, A. Wang, M.A. Green, F. Ferrazza, Appl. Phys. Lett. 73, 1991 (1998). 18. C. Wu, C.H. Crouch, L. Zhao, J.E. Carey, R. Younkin, J.A. Levinson, E. Mazur, R.M. Farrell, P. Gothoskar, A. Karger, Appl. Phys. Lett. 78, 1850 (2001).

19. K. Peng, Y. Xu, Y. Wu, Y. Yan, S.-T. Lee, J. Zhu, Small 1, 1062 (2005).

20. B. Tian, X. Zheng, T.J. Kempa, Y. Fang, N. Yu, G. Yu, J. Huang, C.M. Lieber, Nature 449, 885 (2007)
21. L. Tsakalakos, J. Balch, J. Fronheiser, B.A. Korevaar, O. Sulima, J. Rand, Appl. Phys. Lett. 91, 233117 (2007).

22. L. Hu, G. Chen, Nano Lett. 7, 3249 (2007).

23. O.L. Muskens, J.G. Rivas, R.E. Algra, E.P.A.M. Bakkers, A. Lagendijk, Nano Lett. 8, 2638 (2008).

24. B.M. Kayes, H.A. Atwater, N.S. Lewis, J. Appl. Phys. 97, 114302 (2005).

25. M.D. Kelzenberg, S.W. Boettcher, J.A. Petykiewicz, D.B. Turner-Evans, M.C

Putnam, E.L. Warren, J.M. Spurgeon, R.M. Briggs, N.S. Lewis, H.A. Atwater, Nat. Mater. 9, 239 (2010)

26. O. Gunawan, S. Guha, Sol. Energy Mater. Sol. Cells 93, 1388 (2009).

27. E. Garnett, P. Yang, Nano Lett. 10, 1082 (2010).

28. W.U. Huynh, J.J. Dittmer, A.P. Alivisatos, Science 295, 2425 (2002).

29. M. Law, L.E. Greene, J.C. Johnson, R. Saykally, P. Yang, Nat. Mater. 4, 455 (2005).

30. J.B. Baxter, E.S. Aydil, Appl. Phys. Lett. 86, 053114 (2005)

31. K. Zhu, T.B. Vinzant, N.R. Neale, A.J. Frank, Nano Lett. 7, 3739 (2007).

32. R.A. Pala, J. White, E. Barnard, J. Liu, M.L. Brongersma, Adv. Mater. 34 3504 (2009)

33. H.A. Atwater, A. Polman, Nat. Mater. 9, 205 (2010).

34. O. Stenzel, S. Wilbrandt, A. Stendal, U. Beckers, K. Voigtsberger, C. von Borczyskowski, J. Phys. D 28, 2154 (1995)

35. O. Stenzel, A. Stendal, K. Voigtsberger, C. von Borczyskowski, Sol. Energy Mater. Sol. Cells 37, 337 (1995)

36. M. Westphalen, U. Kreibig, J. Rostalski, H. Lüth, D. Meissner, Sol. Energy Mater. Sol. Cells 6197 (2000).

37. H.R. Stuart, D.G. Hall, Appl. Phys. Lett. 69, 2327 (1996).

38. H.R. Stuart, D.G. Hall, Appl. Phys. Lett. 73, 3815 (1998).

39. H.R. Stuart, D.G. Hall, Phys. Rev. Lett. 80, 5663 (1998).

40. B.J. Soller, D.G. Hall, J. Opt. Soc. Am. A 18, 2577 (2001)

41. B.J. Soller, H.R. Stuart, D.G. Hall, Opt. Lett. 26, 1421 (2001).

42. B.P. Rand, P. Peumans, S.R. Forrest, J. Appl. Phys. 96, 7519 (2004).

43. A.J. Morfa, K.L. Rowlen, T.H. Reilly III, M.J. Romero, J. van de Lagemaat, Appl. Phys. Lett. 92, 013504 (2008).

44. D.M. Schaadt, B. Feng, E.T. Yu, Appl. Phys. Lett. 86, 063106 (2005)

45. S.H. Lim, W. Mar, P. Matheu, D. Derkacs, E.T. Yu, J. Appl. Phys. 101, 104309 (2007).

46. P. Matheu, S.H. Lim, D. Derkacs, C. McPheeters, E.T. Yu, Appl. Phys. Lett. 93, 113108 (2008).

47. D. Derkacs, S.H. Lim, P. Matheu, W. Mar, E.T. Yu, Appl. Phys. Lett. 89, $093103(2006)$

48. K.R. Catchpole, S. Pillai, J. Appl. Phys. 100, 044504 (2006).

49. S. Pillai, K.R. Catchpole, T. Trupke, M.A. Green, J. Appl. Phys. 101, 093105 (2007).

50. D. Derkacs, W.V. Chen, P.M. Matheu, S.H. Lim, P.K.L. Yu, E.T. Yu, Appl. Phys. Lett. 93, 091107 (2008).

51. V.E. Ferry, L.A. Sweatlock, D. Pacifici, H.A. Atwater, Nano Lett. 8, 4391 (2008)

52. K. Nakayama, K. Tanabe, H.A. Atwater, Appl. Phys. Lett. 93, 121904 (2008).

53. S.H. Lim, D. Derkacs, E.T. Yu, J. Appl. Phys. 105, 073101 (2009).

54. C.O. McPheeters, C.J. Hill, S.H. Lim, D. Derkacs, D.Z. Ting, E.T. Yu, J. Appl. Phys. 106, 056101 (2009).

55. V.E. Ferry, M.A. Verschuuren, H.B.T. Li, E. Verhagen, R.J. Walters, R.E.I. Schropp, H.A. Atwater, A. Polman, Opt. Express 18, A237 (2010).

56. Z.F. Yu, A. Raman, S.H. Fan, Proc. Natl. Acad. Sci. U.S.A. 107, 17491 (2010). 57. Z.F. Yu, A. Raman, S. Fan, Opt. Express 18, A366 (2010).

58. J.H. Karp, E.J. Tremblay, J.E. Ford, Opt. Express 18, 1122 (2010).

59. W.G. Van Sark, K.W. Barnham, L.H. Slooff, A.J. Chatten, A. Büchtemann, A. Meyer, S.J. Mc.Cormack, R. Koole, D.J. Farrell, R. Bose, E.E. Bende, A.R. Burgers, T. Budel, J. Quilitz, M. Kennedy, T. Meyer, S.H. Wadman, G.P. van Klink, G. van Koten, A. Meijerink, D. Vanmaekelbergh, Opt. Express 16, 21773 (2008) 60. U.S. Energy Information Administration, Annual Energy review: http://www. eia.doe.gov/emeu/aer/

61. H. Atwater, MRS Bull. 36, 57 (2011).

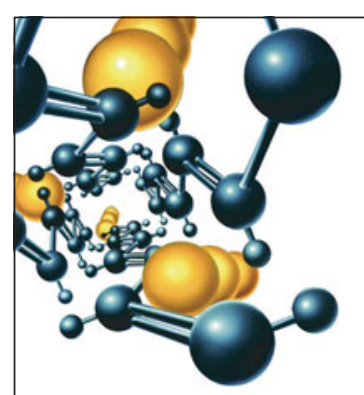

Organic Microelectronics \& Optoelectronics Workshop VII

July 18-20, 2011

Westin San Francisco Market Street, San Francisco, CA

For information on this Workshop, including speakers, schedules, lodging and registration visit www.mrs.org/org-micro7 